\title{
Assembly of Silicon Micro-parts with Steel Spindles Using Low-Temperature Soldering
}

\author{
Laurenz Notter and Jacques Jacot \\ Laboratoire de Production Microtechnique (LPM) \\ École polytechnique fédérale de Lausanne (EPFL) \\ CH-1015 Lausanne, Suisse \\ laurenz.notter@a3.epfl.ch \\ http://lpm.epfl.ch
}

\begin{abstract}
A new assembly method for silicon micro-parts with non-planar steel parts is proposed: low-temperature soldering. Existing techniques for micromechanical assemblies are analyzed and compared to the proposed method, the method is explained and validated on an existing product using functional tests. Performances of the reference method (adhesive bonding) are not yet fully reached, but the results are close and partially fulfil the specifications of the current production.
\end{abstract}

Keywords: Assembly, micro-part, silicon, steel, low-temperature soldering, surface tension, micro-mechanics.

\section{Introduction}

The potential for producing complex micro-mechanical planar pieces from silicon using clean-room fabrication processes has been known for some time. In the field of micro-mechanics - we consider characteristic dimensions between $100 \mu \mathrm{m}$ and $1 \mathrm{~mm}$ and dimensional precision in the micrometer-range - such silicon pieces can be very useful because of their high mechanical strength, their low geometric tolerances, low weight and the parallel fabrication method. Even though the production of silicon micro-pieces is possible using currently available technologies, their application is limited due to three main problems: the maximum achievable thickness, the limited feasibility of multi-layer designs and their integration into out-of-plane assemblies.

In this paper we describe an assembly method that permits to circumvent some of these limitations and compare it to existing techniques using functional tests.

Context and Motivation. Our analysis is part of a research project with industrial partners active in the watch industry and is funded by the Swiss government ${ }^{1}$. The research is guided by the need for a combination of silicon micro-parts with steel pieces so as to permit an integration into existing assemblies while keeping the costs at a viable level for an industrial production. For this reason, we focus on a typical

1 Commission for Technology and Innovation CTI.

S. Ratchev (Ed.): IPAS 2014, IFIP AICT 435, pp. 8-14, 2014.

(C) IFIP International Federation for Information Processing 2014 
assembly problem in this domain: the escape wheel and its spindle. In Fig. 1 one of the realized assemblies is pictured.

Differentiation from MEMS. Unlike the widely researched field of MEMS, which operate on smaller scales, this paper focuses on the integration of silicon micro-parts into existing assemblies such as mechanical movements that are on the scale of some centimeters.

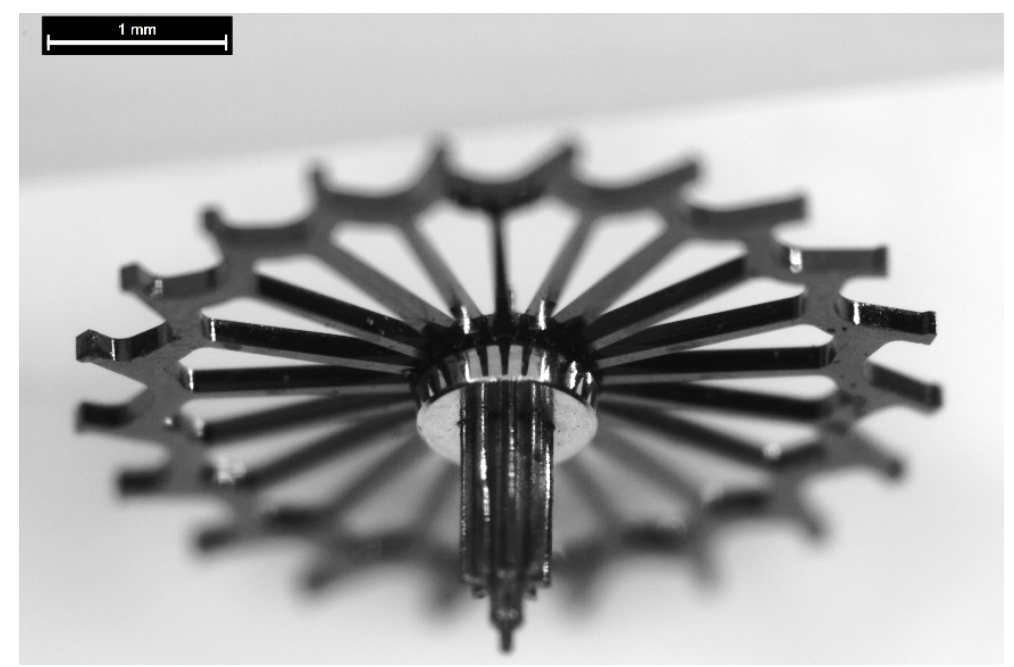

Fig. 1. An escape wheel assembly after low-temperature soldering and torque test

Fabrication of High-precision Mechanical Parts Made of Silicon. Clean-room fabrication processes such as (deep) reactive ion etching (RIE or DRIE) have been used to produce high-precision micro-mechanical parts from silicon wafers such as gears, springs [2,5] and more complex pieces such as multi-level components [3] or rotors for micromotors [4].

Compared to metal parts fabricated using techniques such as fine-blanking, mechanical silicon parts offer greater liberty for the design of their shape while guaranteeing sub-micrometer dimensional precision. As silicon has a lower density and a higher tensile strength than stainless steel (see [6]), mechanical applications benefit from the possibility of lightweight yet resistant designs.

\section{Established Assembly Methods for Silicon Micro-parts with other Materials}

The integration of mechanical silicon parts into assemblies using other materials such as steel or nickel is still a challenge. Conventional (macroscopic) mechanical assembly 
methods rely heavily on properties common for most metals such as plastic deformation (screwing, press-fitting) thermal diffusion and dilatation (soldering, welding, riveting). As these properties are weak or non-existent for silicon and for its native oxide, most conventional techniques are not easily applicable.

In this study we focus on the combination of planar silicon micro-parts with metallic out-of-plane parts such as spindles and compare the established assembly technique to our proposed method of low-temperature soldering.

Adhesive Bonding. A common assembly method for joining silicon and steel parts is adhesive bonding using one- or two component glues. Even though this method yields good results, we seek to propose an alternative because of the sometimes limited chemical stability of the joint. This concerns mainly the exposition to changing ambient humidity and temperature as well as the degradation of the adhesive under ultraviolet radiation. Especially the long term ( $>10$ years) mechanical resistance is difficult to assess. Also, cycle times are relatively long due to the time needed for polymerization $^{2}$ and the assembly process is not easy to get under control.

Press-fitting with Compliant Structures. Press-fitting of structured silicon microparts has been shown to be a viable solution to our assembly problem but is protected by at least one patent [1]. This solution is applicable for low torque transmissions and small axial loads but it has also been proposed to combine a pre-assembly by press-fit with adhesive bonding or soldering to "lock" the assembly and thus overcome these limitations. To our knowledge this method has not yet been commercialized.

Low-temperature Soldering. Low-temperature soldering of silicon to steel is a technique that is very similar in application to adhesive bonding (when a convection oven and solder paste is used). Some of the drawbacks of adhesive bonding can be avoided with low-temperature soldering as soldered joints exhibit generally higher chemical stability as well as a resistance to humidity and ultraviolet radiation.

\subsection{Comparison of Methods}

We propose a qualitative overview of the above mentioned assembly methods that is based on criteria established by a functional analysis of the escapements of mechanical movements, as they are widely used in the Swiss watch industry. In addition to the precise geometric tolerances and mechanical resistance, a long lifespan is required. As the materials of the pieces to assemble are inert, the lifespan is limited by the stability of the joint. Wear may be caused by frequent exposure of the product to rapidly changing ambient atmospheric conditions such as temperature, UV radiation and humidity.

2 Usually in the range of some minutes to several hours, depending on the type of adhesive. 
Table 1. Overview of assembly methods for silicon wheels with steel spindles

\begin{tabular}{llll}
\hline Criteria & $\begin{array}{l}\text { Adhesive } \\
\text { bonding }\end{array}$ & $\begin{array}{l}\text { Press-fitting } \\
\text { with compliant } \\
\text { structures }\end{array}$ & $\begin{array}{l}\text { Low-temperature } \\
\text { soldering }\end{array}$ \\
\hline Prototyping possible? & yes & no & (yes) $)^{3}$ \\
Mechanical resistance & medium & low & medium \\
Chemical stability & medium & high & high \\
Disassembly possible? & no & yes & no \\
Cycle time & medium & short & short \\
\hline
\end{tabular}

Given the qualitative overview of Table 1 it is reasonable to explore the potential of low-temperature soldering. We expect similar performances to adhesive bonding while potentially improving the long-term reliability of the assembly and reducing costs due to shorter cycle times.

\section{Assembly of Silicon Escape Wheels with Their Spindles}

One of the assemblies realized using low-temperature soldering is depicted in Fig. 1 and its schematic representation can be found in Fig. 2. To qualify the result of our proposed method we compare our samples to an existing product that is assembled by adhesive bonding: the escape wheel with its steel spindle. We also use the same tests and criteria, which are used to qualify the ongoing production, for evaluating our assembly method.

The angular deviation from perpendicularity $\beta$ and the concentricity error $d$ are used to qualify the geometry, whereas the mechanical resistance is characterized by the maximum transmissible torque $T_{\max }$. The visual aspect is very important for this type of assembly, but its objective measurement goes beyond the scope of this paper.

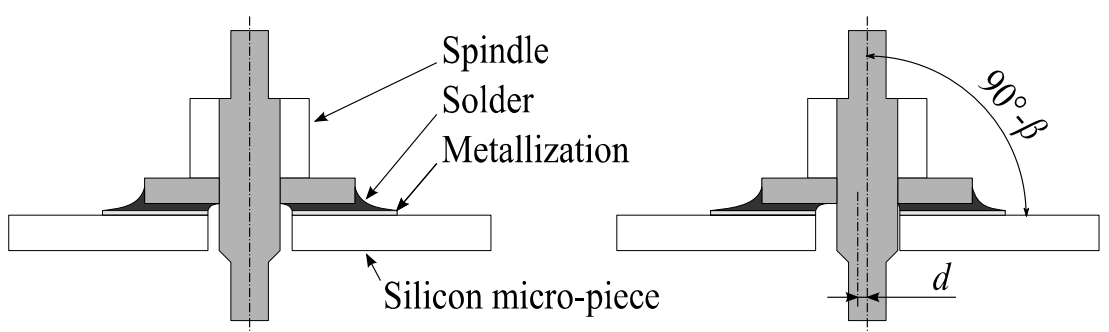

Fig. 2. Schematic representation of the assembled escape wheel with an indication of the concentricity error (distance $d$ ) and the deviation from perpendicularity (angle $\beta$ )

3 A metallization layer can easily be applied to the whole surface of a piece, but as soon as localized metallization is needed, prototyping is not possible anymore. 
Preliminary Condition for Soldering of Silicon Micro-parts. Silicon forms very stable oxides in ambient atmosphere that cannot be removed using simple cleaning processes or fluxes. A convenient solution is to deposit a metallization $\mathrm{Ti}-\mathrm{Ni}-\mathrm{Au}^{4}$ on the silicon substrate, so that we can rely on decades of experience in assembly of metallic surfaces by soldering.

Silicon oxides are not wetted by molten metal, this implies that a localized metallization can be used to control the precise location of the solder joint. Depending on the geometry of the pieces to assemble, this property can be used to hide the solder joint.

\section{$4 \quad$ Soldering Process}

As is succinctly described by Humpston and Jacobson: "Soldering and brazing involve using a molten filler metal to wet the mating surfaces of a joint, with or without the aid of a fluxing agent, leading to the formation of metallurgical bonds between the filler and the respective components. In these processes, the original surfaces of the components are 'eroded' by virtue of the reaction occurring between the molten filler metal and the solid components, but the extent of this 'erosion' is usually at the microscopic level (<100 $\mu \mathrm{m}$, or $4000 \mu \mathrm{in}$.)." [7]

In this section we retrace the different steps of the process and discuss particularities of our application. The usual preconditions for solder joints apply: the surfaces to join should permit a wetting of the filler metal. During the soldering the temperatures of the pieces and their mating surfaces must stay above the liquidus of the filler metal.

\subsection{Degreasing before Soldering}

A cleaning (degreasing) of the pieces is often necessary before the soldering takes place. The escape wheels come from the cleanroom and therefore they are already as clean as they can reasonably get. The spindles have been cleaned using an ultrasonic bath with filtered isopropanol.

\subsection{Positioning of Pieces for the Assembly}

Following the cleaning, the spindle is inserted in a fixture that assures its relative position to the wheel. Solder and flux are deposited on the surface to join and the wheel is positioned on top of it (see Fig. 2 for a schematic representation).

Mechanical Precision of Assembly. We use two geometrical criteria to evaluate the functionality of the assembly: the perpendicularity and the concentricity between spindle and wheel. Depending on the design of the spindle, perpendicularity can be achieved "by default" as the surface tensions of the liquid solder will equilibrate the

4 Titanium is used for adhesion on the silicon substrate, the nickel-layer serves as interface for the solder joint, whereas the gold is applied on top to prevent premature oxidation of the underlying nickel. 
distance between the metallized silicon and the mating surface of the steel spindle. The concentricity error is limited by the clearance between the diameter of the spindle and the inner diameter of the wheel.

Quantity of Metal for Solder Joint. In assemblies of this scale it is not trivial to deposit the optimum quantity of filler metal at the joint. During our research we have qualified several deposition methods but finally the most basic ones - solder paste dispensing and solder preforms - have proven to be sufficient for this application.

\subsection{Soldering Operation}

The fixture with the pre-assembled pieces is heated using a convection oven with control of the temperature profile. We focused on low-temperature solder alloys so that temperatures stay below $200^{\circ} \mathrm{C}$, thereby avoiding any coloration of steel that may happen at or above that point due to additional surface oxidation.

Removal of Surface Oxides. Chemical agents that remove surface oxides (fluxes) are commonly used. Sometimes they are complemented by an inert atmosphere for reducing supplementary surface oxidation during the heating, for example using nitrogen. In our case, the removal of surface oxides concerns mainly the steel piece and the filler metal itself.

\subsection{Cleaning after Soldering}

Depending on the type of flux used, the subsequent cleaning-process is more or less complex. The use of water-soluble fluxes has been found to be sufficient and therefore cleaning is done in aqueous ultrasonic baths.

\section{$5 \quad$ Results}

In section 3 we explained the design of the escape wheel assembly that has been used for comparative tests. In Table 2 the geometrical errors $\beta$ (deviation from perpendicularity) and $d$ (concentricity) as well as the maximum transmissible torque $T_{\max }$ are reported for two assembly methods. Target values are also reproduced to allow a more comprehensive comparison.

Table 2. Comparison of two methods by measuring the performances of the assemblies. For every variable the mean value and the estimator of the standard deviation are indicated.

\begin{tabular}{lllllll}
\hline Method & $\bar{\beta}$ & $s(\bar{\beta})$ & $\bar{d}$ & $s(\bar{d})$ & $\bar{T}_{\max }$ & $s\left(\bar{T}_{\max }\right)$ \\
\hline Adhesive bonding & $0.3^{\circ}$ & $0.18^{\circ}$ & $6 \mu \mathrm{m}$ & $1.8 \mu \mathrm{m}$ & $4.5 \mathrm{mNm}$ & $0.6 \mathrm{mNm}$ \\
Low-temperature & $0.7^{\circ}$ & $0.45^{\circ}$ & $6 \mu \mathrm{m}$ & $5.1 \mu \mathrm{m}$ & $3.4 \mathrm{mNm}$ & $1.2 \mathrm{mNm}$ \\
soldering & $<0.5^{\circ}$ & - & $<12 \mu \mathrm{m}$ & - & $>2 \mathrm{mNm}$ & - \\
Target value & & &
\end{tabular}


Discussion of Results. Our proposed alternative approach does not yet fully reach the performances of the current assembly method, however the results are close enough to be very promising. Two out of three criteria are already above the acceptance limits of the ongoing production, and we have evidence that a significant improvement for the third one is possible.

\section{Conclusion}

The presented approach of low-temperature soldering for the assembly of silicon micro-parts with steel spindles is a valid alternative to conventional adhesive bonding. The comparison with the current assembly method shows that the performances are close enough to lead to an equivalence of the methods in the near future and that an industrialization is of interest.

Identified Subjects for Future Research. Long term comparative tests between adhesive bonding and low-temperature soldering should be carried out, notably concerning the mechanical resistance. Also, it seems that the formation of intermetallic compounds in the boundary layers has a major influence on the transmissible efforts and the resilience to mechanical shocks. Therefore it would be interesting to characterize mechanical performances in function of used alloys, fluxes and microstructure of the interfaces.

Acknowledgements. We thank our partners Mimotec SA and Sigatec SA as well as the members of our project team for their efforts and the pleasant cooperation.

\section{References}

1. Conus, T., Verardo, M., Kohler, F., Saglini, I., Jurin, A.C., Jacot, J., Perret-Gentil, M.: Assembly of a part not comprising a plastic range (2012)

2. Guckel, H., Skrobis, K.J., Christenson, T.R., Klein, J., Han, S., Choi, B., Lovell, E.G.: Fabrication of assembled micromechanical components via deep x-ray lithography, pp. 74-79 (1991)

3. Huber, R., Conrad, J., Schmitt, L., Hecker, K., Scheurer, J., Weber, M.: Fabrication of multilevel silicon structures by anisotropic deep silicon etching. Microelectronic Engineering 67-68, 410-416 (2003); Proceedings of the 28th International Conference on Micro- and Nano-Engineering

4. Mehregany, Gabriel, K., Trimmer, W.: Micro gears and turbines etched from silicon. Sensors and Actuators 12(4), 341-348 (1987)

5. Um, D.: Micro scale silicon dioxide gear fabrication by bulk micromachining process (2010)

6. Petersen, K.E.: Silicon as a mechanical material. Proceedings of the IEEE 70(5), 420-457 (1982)

7. Humpston, G., Jacobson, D.M.: Principles of Soldering. ASM International (2004) 